\title{
LMI-Based Stability Criteria for Discrete-Time Neural Networks with Multiple Delays
}

\author{
Hui Xu ${ }^{1,2}$ and Ranchao $W u^{1}$ \\ ${ }^{1}$ School of Mathematics, Anhui University, Hefei 230039, China \\ ${ }^{2}$ Department of Public Teaching, Anhui Business Vocational College, Hefei 230041, China
}

Correspondence should be addressed to Ranchao Wu; rcwu@ahu.edu.cn

Received 17 March 2013; Accepted 26 May 2013

Academic Editor: Wen Xiu Ma

Copyright (C) 2013 H. Xu and R. Wu. This is an open access article distributed under the Creative Commons Attribution License, which permits unrestricted use, distribution, and reproduction in any medium, provided the original work is properly cited.

\begin{abstract}
Discrete neural models are of great importance in numerical simulations and practical implementations. In the current paper, a discrete model of continuous-time neural networks with variable and distributed delays is investigated. By Lyapunov stability theory and techniques such as linear matrix inequalities, sufficient conditions guaranteeing the existence and global exponential stability of the unique equilibrium point are obtained. Introduction of LMIs enables one to take into consideration the sign of connection weights. To show the effectiveness of the method, an illustrative example, along with numerical simulation, is presented.
\end{abstract}

\section{Introduction}

During the past decades, various types of neural networks have been proposed and investigated intensively, since they play important roles and have found successful applications in fields such as pattern recognition, signal and image processing, nonlinear optimization problems, and parallel computation. The dynamical behaviors in neural models, such as the existence and their asymptotic stability of equilibria, periodic solutions, bifurcations, and chaos, have been the most active areas of research and have been extensively explored over the past years [1-22].

Due to the finite transmission speed of signals among neurons, time delays in interactions between neurons frequently happen and will cause complex dynamics in neural networks [6]; so it is necessary to introduce time delays into the neural models. So far, discrete, time-varying, and distributed delays have been, respectively, introduced to describe the dynamics of neural networks, and various sufficient conditions ensuring the stability have been given.

Note that in numerical simulation and practical implementations, discretization of continuous-time models is necessary and of great importance. On the other hand, the dynamics of discrete-time neural networks could be quite different from those of continuous versions and will display much more complicated behaviors. So it is of great theoretical and practical significance to study the dynamics of discrete neural models. For discrete models, such as discrete Hopfield, bidirectional associate memory, and Cohen-Grossberg neural networks, several authors [1,7-22] have studied the existence and exponential stability of equilibria and periodic solutions.

In this paper, a discrete model with both variable and distributed time delays is introduced. By Lyapunov stability theory and linear matrix inequality (LMI) technique, sufficient conditions ensuring the existence and globally exponential stability of a unique equilibrium point are obtained. To show the effectiveness of our results, an illustrative example along with numerical simulation is presented. To our best knowledge, such general models have been seldom touched upon in the existing literatures. As we see, the obtained conditions are easy to verify. Furthermore, introduction of LMIs enables us to take into consideration the sign of connection weights. In contrast, sufficient conditions, for instance, in [7-12], depend on the absolute values of connection weights. That will ignore the differences between neuronal excitatory and inhibitory effects. 


\section{Preliminaries}

Set $Z$ to be the set of integers and $Z^{+}$the set of nonnegative integers; let $\mathbf{N}(a, b)$ represent the set of integers between $a$ and $b$ with $a \leq b, a, b \in Z$, namely, $\mathbf{N}(a, b)=\{a, a+1, \ldots, b\}$.

Consider the discrete-time neural networks with both variable and distributed delays:

$$
\begin{aligned}
x_{i}(n+1)= & a_{i} x_{i}(n)+\sum_{j=1}^{m} b_{i j} f_{j}\left(x_{j}(n-k(n))\right) \\
& +\sum_{j=1}^{m} c_{i j} \sum_{p=1}^{\infty} \mathscr{K}_{j}(p) g_{j}\left(x_{j}(n-p)\right)+I_{i},
\end{aligned}
$$

with initial values

$$
x_{i}(l)=\phi_{i}(l), \quad l \in \mathbf{N}(-\infty, 0),
$$

where $x(n)=\operatorname{col}\left(x_{1}(n), \ldots, x_{m}(n)\right) \in R^{m}, x_{i}(n)$ are the states of the $i$ th neuron at time $n ; a_{i} \in(0,1)$ represents the rate with which the $i$ th neuron resets its potential when isolated from others; $b_{i j}$ and $c_{i j}$ weigh the strengths of the $j$ th unit on the $i$ th unit; $f_{j}$ and $g_{j}$ are the nonlinear activation functions of the neurons; $k(n)$ denotes the transmission delay along the axon of the $j$ th unit; $\mathscr{K}_{j}(p) \geq 0$ is the delay kernel; $I_{i}$ is the external input on the $i$ th neuron at time $n$; the initial value functions $\phi_{i}(l)$ are bounded $\mathbf{N}(-\infty, 0), i=1, \ldots, m$.

To investigate stability of system (1), make further assumptions:

(H1) suppose that $b_{i j}, c_{i j}, I_{i} \in R$, for $i=1, \ldots, m$, and $0 \leq$ $k(n) \leq k$ for $n \in \mathbf{N}(0, \infty)$, with $k$ being constant;

(H2) suppose that $\mathscr{K}_{j}(p) \geq 0, \sum_{p=1}^{\infty} \mathscr{K}_{j}(p)=1$, and $\sum_{p=1}^{\infty} \mathscr{K}_{j}(p) v^{p}<+\infty$, for some $v>1$, all $i, j=$
$1, \ldots, m ;$

(H3) assume that functions $f_{j}$ and $g_{j}$ are bounded and satisfy

$$
f_{j}^{-} \leq \frac{f_{j}(\xi)-f_{j}(\eta)}{\xi-\eta} \leq f_{j}^{+}, \quad g_{j}^{-} \leq \frac{g_{j}(\xi)-g_{j}(\eta)}{\xi-\eta} \leq g_{j}^{+},
$$

for any $\xi, \eta \in R$, where $f_{j}^{+}, f_{j}^{-}, g_{j}^{+}$, and $g_{j}^{-}$are some constants and can be positive, negative, or zero, $j=1, \ldots, m$. So they are less restrictive than sigmoid activation functions and Lipschitz-type ones.

For any $\phi=\left(\phi_{1}, \ldots, \phi_{m}\right)$, a solution of systems (1) and (2) is a vector-valued function $x: Z^{+} \rightarrow R^{m}$ satisfying system (1) and initial conditions (2) for $n \in Z^{+}$. In this paper, it is always assumed that neural model (1) admits a solution represented by $x(n, \phi)$ or simply $x(n)$. Since the activation functions $f_{j}, g_{j}$ are bounded, it is not difficult to check that system (1) has at least one equilibrium point by Brouwer's fixed point theorem. So with loss of generality, assume that $f_{j}(0)=g_{j}(0)=0$; that is, $x=0$ is an equilibrium point. Throughout the paper, denote

$$
\begin{gathered}
\|x(n)\|^{2}=\sum_{i=1}^{m}\left|x_{i}(n)\right|^{2}, \\
k_{M}=\sup _{n}\{k(n)\}, \quad k_{m}=\inf _{n}\{k(n)\}, \\
F_{1}=\operatorname{diag}\left\{f_{1}^{+} f_{1}^{-}, \ldots, f_{m}^{+} f_{m}^{-}\right\}, \\
F_{2}=\operatorname{diag}\left\{f_{1}^{+}+f_{1}^{-}, \ldots, f_{m}^{+}+f_{m}^{-}\right\}, \\
G_{1}=\operatorname{diag}\left\{g_{1}^{+} g_{1}^{-}, \ldots, g_{m}^{+} g_{m}^{-}\right\}, \\
G_{2}=\operatorname{diag}\left\{g_{1}^{+}+g_{1}^{-}, \ldots, g_{m}^{+}+g_{m}^{-}\right\}, \\
\left.\left.B=\operatorname{diag}\left\{a_{1}, \ldots, a_{m}\right\}, \quad C b_{i j}\right)_{m \times m}, \quad C=\left(c_{i j}\right)_{m \times m}\right\} \\
f=\left(f_{1}, \ldots, f_{m}\right)^{T}, \quad g=\left(g_{1}, \ldots, g_{m}\right)^{T}, \\
\mathscr{K}=\operatorname{diag}\left(\mathscr{K}_{1}, \ldots, \mathscr{K}_{m}\right) .
\end{gathered}
$$

System (1) can be rewritten into the form

$$
\begin{aligned}
x(n+1)= & A x(n)+B f(x(n-k(n))) \\
& +C \sum_{p=1}^{\infty} \mathscr{K}(p) g(x(n-p)) .
\end{aligned}
$$

Definition 1. The equilibrium point $x=0$ of system (1) is globally exponentially stable if there exist constants $\eta>1$ and $C^{*}>0$ such that for any solution $x(n, \phi)$ of system (1) with initial conditions $\phi$, it holds that

$$
\|x(n, \phi)\|^{2} \leq C^{*} \eta^{-n} \sup _{l \in \mathbf{N}(-\infty, 0)}\|\phi(l)\|^{2}, \quad \forall n \in Z^{+}
$$

\section{Exponential Stability of Equilibrium Points}

By Lyapunov stability theory and LMI technique, the global exponential stability of the equilibrium point is established. Clearly, if $x=0$ is exponentially stable, the equilibrium point is unique. Now we will investigate the exponential stability of the origin.

Theorem 2. Suppose that (H1)-(H3) hold and further there exist a number $v \geq \eta>1$, positive definite matrix $P$, $\Sigma=\operatorname{diag}\left\{e_{1}, \ldots, e_{m}\right\}$, and semipositive diagonal matrices 
$U=\operatorname{diag}\left\{u_{1}, \ldots, u_{m}\right\}, V=\operatorname{diag}\left\{v_{1}, \ldots, v_{m}\right\}$, and $S=$ $\operatorname{diag}\left\{s_{1}, \ldots, s_{m}\right\}$, such that

W

$$
=\left[\begin{array}{cccccc}
\Phi_{11} & 0 & A P B+U F_{2} & 0 & V G_{2} & A P C \\
* & -2 S F_{1} & 0 & S F_{2} & 0 & 0 \\
* & * & \Phi_{33} & 0 & 0 & 0 \\
* & * & * & \Phi_{44} & 0 & B^{T} P C \\
* & * & * & * & \alpha \Sigma-2 V & 0 \\
* & * & * & * & * & \eta C^{T} P C-\Sigma
\end{array}\right]
$$

$<0$,

where

$$
\begin{gathered}
\Phi_{11}=\eta A P A-P-2 U F_{1}-2 V G_{1}, \\
\Phi_{33}=\left(k_{M}-k_{m}+1\right) \Sigma-2 U, \\
\Phi_{44}=\eta B^{T} P B-\eta^{-k_{M}} \Sigma-2 S, \\
\alpha=\operatorname{diag}\left\{\alpha_{1}, \ldots, \alpha_{m}\right\}, \quad \alpha_{j}=\sum_{p=1}^{\infty} \mathscr{K}_{j}(p) \eta^{p} .
\end{gathered}
$$

Then the origin of system (1) is exponentially stable.

Proof. Define a Lyapunov functional $V(n)=V\left(x_{1}, \ldots, x_{m}\right)(n)$ as follows:

$$
V(n)=V_{1}(n)+V_{2}(n)+V_{3}(n)+V_{4}(n),
$$

where

$$
\begin{aligned}
& V_{1}(n)=\eta^{n} x^{T}(n) P x(n), \\
& V_{2}(n)=\sum_{s=n-k(n)}^{n-1} \eta^{s} f^{T}(x(s)) \Sigma f(x(s)), \\
& V_{3}(n)=\sum_{r=-k_{M}+2}^{-k_{m}+1} \sum_{s=n+r-1}^{n-1} \eta^{s} f^{T}(x(s)) \Sigma f(x(s)), \\
& V_{4}(n)=\sum_{j=1}^{m} \sum_{p=1}^{\infty} \mathscr{K}_{j}(p) \sum_{s=n-p}^{n-1} \eta^{s+p} g_{j}^{2}\left(x_{j}(s)\right) e_{j} .
\end{aligned}
$$

To investigate the exponential stability of the origin, it is necessary to calculate the difference $\Delta V(n)=V(n+1)-V(n)$ along the trajectory of (6). From (6), we have

$$
\begin{aligned}
\Delta V_{1}(n)=\eta^{n+1} x^{T}(n+1) P x(n+1)-\eta^{n} x^{T}(x) P x(n) \\
=\eta^{n+1}\left[x^{T}(n) A P A x(n)+2 x^{T}(n) A P B f\right. \\
\quad+(x(n-k(n)))+2 x^{T}(n) A P C \phi(x(n)) \\
\quad+f^{T}(x(n-k(n))) B^{T} P B f(x(n-k(n))) \\
\quad+2 f^{T}(x(n-k(n))) B^{T} P C \phi(x(n)) \\
\left.\quad+\phi(x(n))^{T} C^{T} P C \phi(x(n))\right] \\
-\eta^{n} x^{T}(n) P x(n),
\end{aligned}
$$

where $\phi(x(n))=\sum_{p=1}^{\infty} \mathscr{K}(p) g(x(n-p))$. Since $k_{m} \leq k(n) \leq$ $k_{M}$, one obtains

$$
\begin{aligned}
\Delta V_{2}(n)= & \sum_{s=n+1-k(n+1)}^{n} \eta^{s} f^{T}(x(s)) \Sigma f(x(s)) \\
& -\sum_{s=n-k(n)}^{n-1} \eta^{s} f^{T}(x(s)) \Sigma f(x(s)) \\
= & \eta^{n} f^{T}(x(n)) \Sigma f(x(n)) \\
& -\eta^{n-k(n)} f^{T}(x(n-k(n))) \Sigma f \\
& \times(x(n-k(n)))+\sum_{s=n+1-k_{m}}^{n-1} \eta^{s} f^{T}(x(s)) \Sigma f(x(s))
\end{aligned}
$$$$
+\sum_{s=n+1-k(n+1)}^{n-k_{m}} \eta^{s} f^{T}(x(s)) \Sigma f(x(s))
$$$$
-\sum_{s=n+1-k(n)}^{n-1} \eta^{s} f^{T}(x(s)) \Sigma f(x(s))
$$$$
\leq \eta^{n} f^{T}(x(n)) \Sigma f(x(n))
$$

$$
\begin{aligned}
& -\eta^{n-k(n)} f^{T}(x(n-k(n))) \Sigma f \\
& \times(x(n-k(n)))+\sum_{s=n+1-k_{M}}^{n-k_{m}} \eta^{s} f^{T}(x(s)) \Sigma f(x(s)),
\end{aligned}
$$

$\Delta V_{3}(n)=\left(k_{M}-k_{m}\right) \eta^{n} f^{T}(x(n)) \Sigma f(x(n))$ 


$$
\begin{aligned}
& -\sum_{s=n+1-k_{M}}^{n-k_{m}} \eta^{s} f^{T}(x(s)) \Sigma f(x(s)) \\
\Delta V_{4}(n)= & \sum_{j=1}^{m} \sum_{p=1}^{\infty} \mathscr{K}_{j}(p)\left[\eta^{n+p} g_{j}^{2}(x(n))\right. \\
& \left.-\eta^{n} g_{j}^{2}(x(n-p))\right] e_{j} \\
= & \eta^{n} \sum_{j=1}^{m}\left(\sum_{p=1}^{\infty} \mathscr{K}_{j}(p) \eta^{p}\right) g_{j}^{2}(x(n)) e_{j} \\
& -\eta^{n} \sum_{j=1}^{m} \sum_{p=1}^{\infty} \mathscr{K}_{j}(p) g_{j}^{2}(x(n-p)) e_{j} \\
= & \eta^{n} g^{T}(x(n)) \alpha \Sigma g(x(n)) \\
& -\eta^{n} \sum_{j=1}^{m}\left(\sum_{p=1}^{\infty} \mathscr{K}_{j}(p)\right) \sum_{p=1}^{\infty} \mathscr{K}_{j}(p) g_{j}^{2}(x(n-p)) e_{j} \\
\leq & \eta^{n}\left[g^{T}(x(n)) \alpha \Sigma g(x(n))\right. \\
& \left.\quad-\phi^{T}(x(n)) \Sigma \phi(x(n))\right] .
\end{aligned}
$$

Therefore, we have

$$
\begin{aligned}
& \Delta V(n)=\Delta V_{1}(n)+\Delta V_{2}(n)+\Delta V_{3}(n) \\
& \leq \eta^{n}\left[x^{T}(n)(\eta A P A-P) x(n)\right. \\
&+2 x^{T}(n) A P B f(x(n-k(n))) \\
&+2 x^{T}(n) A P C \phi(x(n)) \\
&+\left(k_{M}-k_{m}+1\right) f^{T} \\
& \quad \times(x(n)) \Sigma f(x(n)) \\
&+f^{T}(x(n-k(n)))\left(\eta B^{T} P B-\eta^{-k_{M}} \Sigma\right) \\
& \times f(x(n-k(n))) \\
&+2 f^{T}(x(n-k(n))) B^{T} P C \phi(x(n)) \\
&+g^{T}(x(n)) \alpha \Sigma g(x(n)) \\
&\left.+\phi(x(n))^{T}\left(\eta C^{T} P C-\Sigma\right) \phi(x(n))\right] .
\end{aligned}
$$

From (H3), one has

$$
\begin{array}{r}
\left(f_{j}\left(x_{j}(n)\right)-f_{j}^{+} x_{j}(n)\right)\left(f_{j}\left(x_{j}(n)\right)-f_{j}^{-} x_{j}(n)\right) \leq 0, \\
\left(g_{j}\left(x_{j}(n)\right)-g_{j}^{+} x_{j}(n)\right)\left(g_{j}\left(x_{j}(n)\right)-g_{j}^{-} x_{j}(n)\right) \leq 0, \\
j=1,2, \ldots, m .
\end{array}
$$

Then for $U=\operatorname{diag}\left\{u_{1}, \ldots, u_{m}\right\} \geq 0, V=\operatorname{diag}\left\{v_{1}, \ldots, v_{m}\right\} \geq 0$, and $S=\operatorname{diag}\left\{s_{1}, \ldots, s_{m}\right\} \geq 0$, one has

$$
\begin{aligned}
\Delta V(n) \leq & \Delta V(n)-2 \sum_{j=1}^{m} u_{j}\left(f_{j}\left(x_{j}(n)\right)-f_{j}^{+} x_{j}(n)\right) \\
& \times\left(f_{j}\left(x_{j}(n)\right)-f_{j}^{-} x_{j}(n)\right) \\
& -2 \sum_{j=1}^{m} v_{j}\left(g_{j}\left(x_{j}(n)\right)-g_{j}^{+} x_{j}(n)\right) \\
& \times\left(g_{j}\left(x_{j}(n)\right)-g_{j}^{-} x_{j}(n)\right) \\
& -2 \sum_{j=1}^{m} s_{j}\left(f_{j}\left(x_{j}(n-k(n))\right)-f_{j}^{+} x_{j}(n-k(n))\right) \\
& \times\left(f_{j}\left(x_{j}(n-k(n))\right)-f_{j}^{-} x_{j}(n-k(n))\right) \\
\leq & \xi^{T} W \xi
\end{aligned}
$$

where $\xi=\left(x^{T}(n), x^{T}(n-k(n)), f^{T}(x(n)), f^{T}(x(n-k(n)))\right.$, $\left.g^{T}(x(n)), \phi(x(n))\right)^{T}$. From $W<0$, it follows that $\Delta V(n)<0$. Note that

$$
\begin{aligned}
V(n) \geq & \lambda_{m}(P) \eta^{n}\|x(n)\|^{2}, \\
V(0) \leq & x^{T}(0) P x(0)+\left(k_{M}-k_{m}+1\right) \\
& \times \sum_{s=-k_{M}}^{-1} \eta^{s} f^{T}(x(s)) \sum f(x(s)) \\
& +\sum_{j=1}^{m} e_{j} \sum_{p=1}^{\infty} \mathscr{K}_{j}(p) \sum_{s=-p}^{-1} \eta^{s+p} g_{j}^{2}\left(x_{j}(s)\right) \\
\leq & {\left[\lambda_{M}(P)+\delta_{1} L\left(k_{M}-k_{m}+1+\delta_{2}\right)\right] \sup _{s \in N(-\infty, 0)}\|x(s)\|^{2}, }
\end{aligned}
$$

where $\lambda_{m}(P), \lambda_{M}(P)$ are the minimum and maximum eigenvalues of matrix $P$, respectively, $\delta_{1}=1 /(\eta-1), \delta_{2}=\sum_{j=1}^{m} e_{j} \alpha_{j}$, and $L=\max \left\{\left|f_{j}^{+}\right|,\left|f_{j}^{-}\right|,\left|g_{j}^{+}\right|,\left|g_{j}^{-}\right|\right\}$, so one has

$$
\|x(n, \phi)\|^{2} \leq C^{*} \eta^{-n} \sup _{l \in \mathbf{N}(-\infty, 0)}\|\phi(l)\|^{2}, \quad n \in Z^{+},
$$

where $C^{*}=\lambda_{m}^{-1}(P)\left[\lambda_{M}(P)+\delta_{1} L\left(k_{M}-k_{m}+1+\delta_{2}\right)\right]$. This implies that the equilibrium solution $x=0$ of system (1) is globally exponentially stable. The proof is completed.

Remark 3. By employing LMI (8), the signs of $b_{i j}, c_{i j}$, that is, the differences between neural excitatory and inhibitory interaction, are taken into consideration.

Remark 4. If $\eta=1$, the equilibrium point 0 of system (1) is said to be globally stable. 


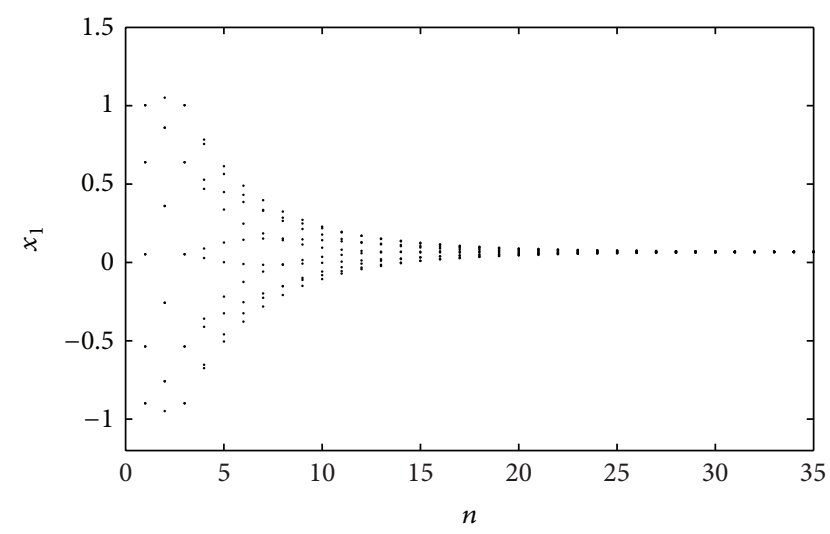

FIgURE 1: Trajectories of $x_{1}(n)$ versus $n$.

\section{Numerical Example}

Next, an illustrative example is given to show the effectiveness of the obtained results. Consider the discrete-time neural model (6) with parameters:

$$
\begin{gathered}
A=\left(\begin{array}{cc}
0.5 & 0 \\
0 & 0.6
\end{array}\right), \quad B=\left(\begin{array}{cc}
0.05 & -0.1 \\
-0.1 & 0.15
\end{array}\right), \\
C=\left(\begin{array}{cc}
0.1 & -0.1 \\
0.05 & 0.15
\end{array}\right), \\
f_{1}(u)=\tanh (2 u), \quad f_{2}(u)=\tanh (-2 u), \\
g_{1}(u)=g_{2}(u)=\arctan u, \\
\mathscr{K}_{1}(p)=\mathscr{K}_{2}(p)=\frac{3}{4^{p}}, \quad k(n)=4+2(-1)^{n},
\end{gathered}
$$

then it is not difficult to see that $F_{1}=G_{1}=0, F_{2}=$ $\operatorname{diag}\{2,-2\}, G_{2}=\operatorname{diag}\{1,1\}, k_{M}=6$, and $k_{m}=2$. Take $\eta=2$, then by solving LMI (8), it has feasible solutions that are $\Sigma=S=V=I$ and

$$
P=\left(\begin{array}{cc}
19.8208 & -1.8309 \\
-1.8309 & 17.0882
\end{array}\right), \quad U=\left(\begin{array}{cc}
2.5213 & 0 \\
0 & 2.5710
\end{array}\right)
$$

so from Theorems 2 , this system admits a unique equilibrium 0 , with all other solutions converging to it exponentially as $n \rightarrow \infty$ see Figures 1 and 2 .

\section{Conclusions}

In the current paper, a class of discrete-time neural networks with both variable and distributed delays has been studied. Using Lyapunov stability and LMI technique, the existence and global exponential stability of the unique equilibrium point have been established. The obtained results are easy to verify, so they will be of practical use for applying discrete neural models.

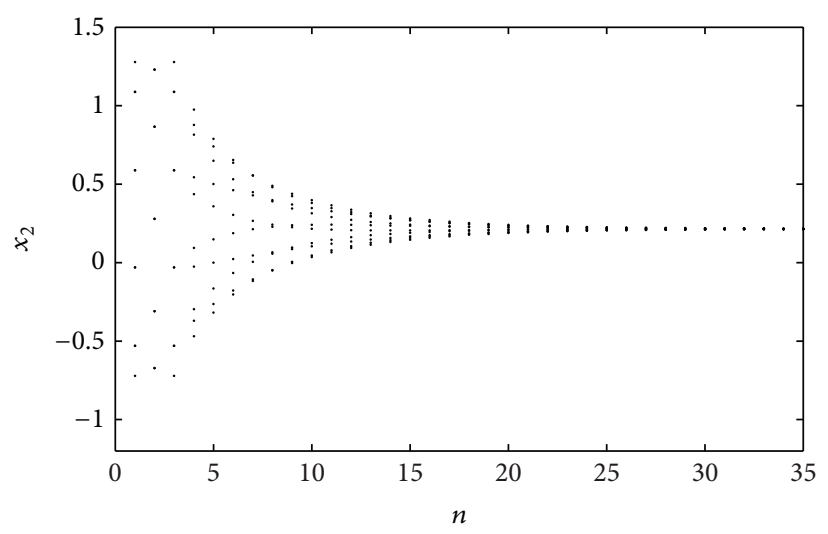

FIGURE 2: Trajectories of $x_{2}(n)$ versus $n$.

\section{Acknowledgments}

This study is supported by the Specialized Research Fund for the Doctoral Program of Higher Education of China (no. 20093401120001), the Natural Science Foundation of Anhui Province (no. 11040606M12), the Natural Science Foundation of Anhui Education Bureau (no. KJ2010A035), the 211 project of Anhui University (no. KJJQ1102).

\section{References}

[1] S. Mohamad and K. Gopalsamy, "Dynamics of a class of discrete-time neural networks and their continuous-time counterparts," Mathematics and Computers in Simulation, vol. 53, no. 1-2, pp. 1-39, 2000.

[2] S. Arik, "An improved global stability result for delayed cellular neural network," IEEE Transactions on Circuits and Systems, vol. 49, no. 8, pp. 1211-1214, 2002.

[3] Z. Wang, Y. Liu, and X. Liu, "On global asymptotic stability of neural networks with discrete and distributed delays," Physics Letters, vol. 345, no. 4-6, pp. 299-308, 2005.

[4] C.-Y. Cheng, K.-H. Lin, and C.-W. Shih, "Multistability and convergence in delayed neural networks," Physica D, vol. 225, no. 1, pp. 61-74, 2007.

[5] S. Mou, H. Gao, J. Lam, and W. Qiang, "A new criterion of delaydependent asymptotic stability for Hopfield neural networks with time delay," IEEE Transactions on Neural Networks, vol. 19, no. 3, pp. 532-535, 2008.

[6] S. I. Niculescu, Delay Effects on Stability: A Robust Approach, Springer, Berlin, Germany, 2001.

[7] J. Liang, J. Cao, and J. Lam, "Convergence of discrete-time recurrent neural networks with variable delay," International Journal of Bifurcation and Chaos, vol. 15, no. 2, pp. 581-595, 2005.

[8] J. Liang, J. Cao, and D. W. C. Ho, "Discrete-time bidirectional associative memory neural networks with variable delays," Physics Letters Section A, vol. 335, no. 2-3, pp. 226-234, 2005.

[9] S. Mohamad, "Global exponential stability in continuoustime and discrete-time delayed bidirectional neural networks," Physica D, vol. 159, no. 3-4, pp. 233-251, 2001.

[10] C. Sun and C.-B. Feng, "Discrete-time analogues of integrodifferential equations modeling neural networks," Physics Letters A, vol. 334, no. 2-3, pp. 180-191, 2005. 
[11] X.-G. Liu, M.-L. Tang, R. Martin, and X.-B. Liu, "Discrete-time BAM neural networks with variable delays," Physics Letters A, vol. 367, no. 4-5, pp. 322-330, 2007.

[12] H. Zhao, L. S. Li Sun, and G. Wang, "Periodic oscillation of discrete-time bidirectional associative memory neural networks," Neurocomputing, vol. 70, no. 16-18, pp. 2924-2930, 2007.

[13] W. He and J. Cao, "Stability and bifurcation of a class of discretetime neural networks," Applied Mathematical Modelling, vol. 31, no. 10, pp. 2111-2122, 2007.

[14] B. Cessac, "A discrete time neural network model with spiking neurons," Journal of Mathematical Biology, vol. 56, no. 3, pp. 311$345,2008$.

[15] Y. Liu, Z. Wang, and X. Liu, "Asymptotic stability for neural networks with mixed time-delays: the discrete-time case," Neural Networks, vol. 22, no. 1, pp. 67-74, 2009.

[16] J. Cao and Q. Song, "Global dissipativity on uncertain discretetime neural networks with time-varying delays," Discrete Dynamics in Nature and Society, vol. 2010, Article ID 810408, 19 pages, 2010.

[17] T. Ensari and S. Arik, "New results for robust stability of dynamical neural networks with discrete time delays," Expert Systems with Applications, vol. 37, no. 8, pp. 5925-5930, 2010.

[18] A. Y. Alanis, E. N. Sanchez, A. G. Loukianov, and E. A. Hernandez, "Discrete-time recurrent high order neural networks for nonlinear identification," Journal of the Franklin Institute, vol. 347, no. 7, pp. 1253-1265, 2010.

[19] Z. Huang, X. Wang, and C. Feng, "Multiperiodicity of periodically oscillated discrete-time neural networks with transient excitatory self-connections and sigmoidal nonlinearities," IEEE Transactions on Neural Networks, vol. 21, no. 10, pp. 1643-1655, 2010.

[20] C. Li, S. Wu, G. G. Feng, and X. Liao, "Stabilizing effects of impulses in discrete-time delayed neural networks," IEEE Transactions on Neural Networks, vol. 22, no. 2, pp. 323-329, 2011.

[21] O. Faydasicok and S. Arik, "Robust stability analysis of a class of neural networks with discrete time delays," Neural Networks, vol. 29-30, pp. 52-59, 2012.

[22] Y. Li and C. Wang, "Existence and global exponential stability of equilibrium for discrete-time fuzzy BAM neural networks with variable delays and impulses," Fuzzy Sets Systems, vol. 217, pp. 62-79, 2013. 


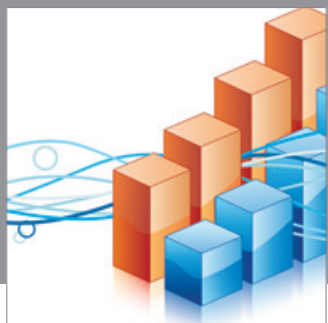

Advances in

Operations Research

mansans

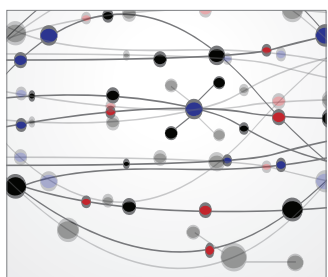

The Scientific World Journal
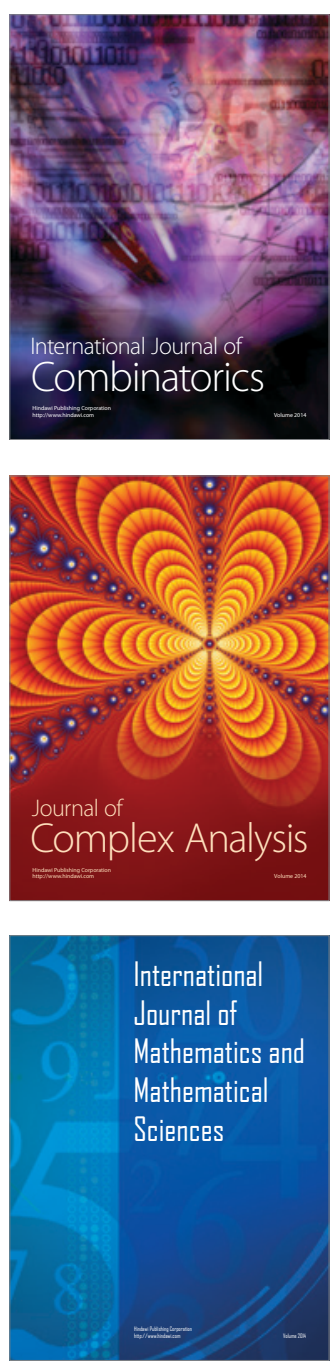
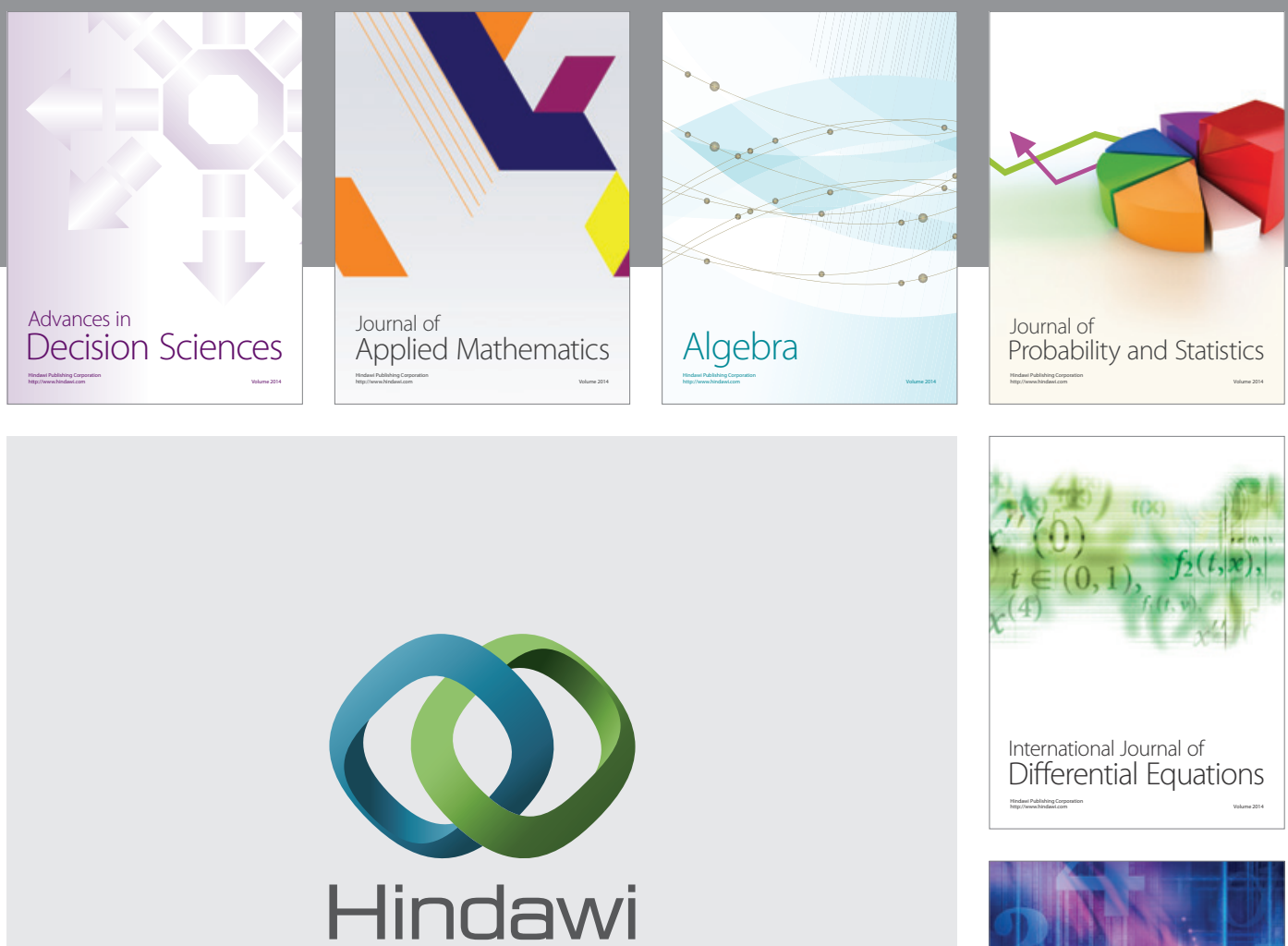

Submit your manuscripts at http://www.hindawi.com
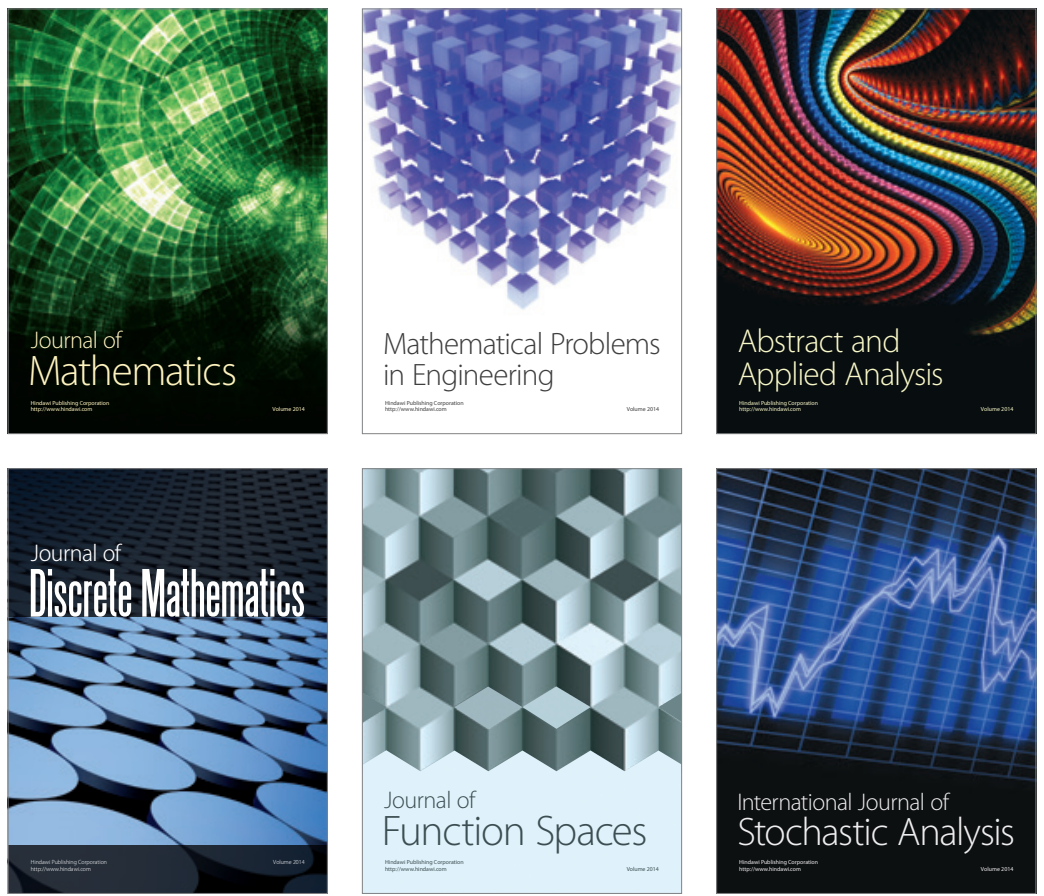

Journal of

Function Spaces

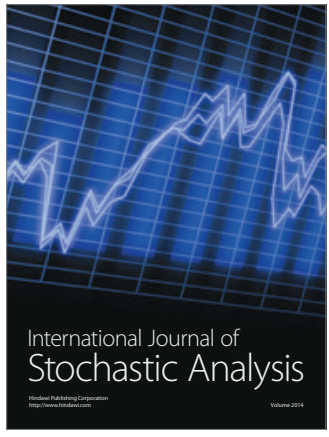

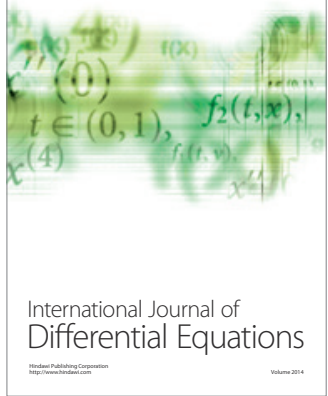
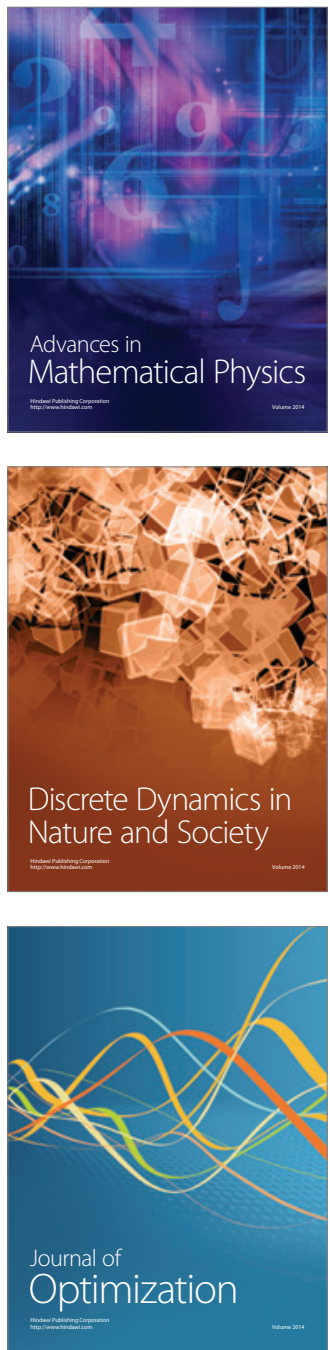